\title{
3D Reconstruction of a Subcomplex of NADH-ubiquinone-oxidoreductase (Complex I) from Yarrowia lipolytica.
}

\author{
M. Radermacher*, T. Ruiz*, D. J. Fowler*, L. Yu*, S. Dröse**, S. Krack**, S. Kerscher**, \\ V. Zickermann**, U. Brandt** \\ * University of Vermont, College of Medicine, Dept. Molecular Physiology and Biophysics, \\ 149 Beaumont Ave, Burlington, VT \\ ** Johann Wolfgang Goethe University, Medical School, Centre for Biological Chemistry, \\ Theodor-Stern-Kai 7, 60590 Frankfurt am Main, Germany
}

Complex I is the first and largest enzyme in the respiratory chain located in the inner mitochondrial membrane and in the cytoplasmic membrane of many bacteria [1]. In the process of oxidizing NADH, Complex I translocates 4 protons across the membrane. This is followed by additional proton translocation by complexes III and IV. The resulting membrane potential powers ATP production by the F-ATPase. The minimal bacterial complex I contains 14 different subunits that are conserved throughout species. Eukaryotic complex I contains more than 26 additional (accessory) subunits. Complex I is L-shaped with a membrane arm and a peripheral arm that contains the NADH binding site and reaches into the mitochondrial matrix. The membrane arm contains 7 conserved subunits, ND1ND4,ND4L,ND5 and ND6. ND2, ND4 and ND5 have sequence homology to certain antiporters [2] and are the putative subunits for active proton translocation [3]. Here we have analyzed the structure of a subcomplex of $Y$. lipolytica complex I, created by the deletion of the accessory subunit NB8M located in the membrane arm with a molecular mass of $11 \mathrm{kDa}$. This resulted in the assembly of a subcomplex of molecular mass of approximately $680 \mathrm{kDa}$ lacking the central subunits ND4, and ND5 [4].This complex showed reduced electron transfer activity $(30 \%)$ and a reduced stoichiometry of proton pumping $(\sim 50 \%)$.

The 3D structure was determined by single particle reconstruction from electron micrographs using the Random Conical reconstruction Technique (RCT)[5]. The sample was prepared on carbon coated grids, deep stain embedded [6,7] in NanoW (Nanoprobes). Tilt pairs were recorded at a nominal magnification of $67 \mathrm{KX}$ with a $55^{\circ}$ tilt angle. 68 tilt pairs were digitized with a final calibrated pixel size of $3.14 \AA$. The microscope transfer function was corrected for defocus and astigmatism in both $0^{\circ}$ - and tilt-images as described in [8]. The $0^{\circ}$ images were first centered and then aligned by iterations of multireference alignments and classifications, using correspondence analysis and classification as the last step. All rotation/ translation alignments were carried out using the simultaneous translation/rotation alignments based on Radon transforms [9]. 3D reconstructions were calculated from the 10 final classes. (Fig.2). The differences between the 10 reconstructions were mainly differences in preferred orientation, which showed that the sample was homogeneous and allowed us to merge all 10 classes into a single reconstruction. The final reconstruction from 10586 images has a resolution of $23 \AA$ determined by Fourier Shell Correlation with a cutoff of 0.3 [10].

A comparison with our earlier 3D structure of the complete complex I [11] and recent X-raycrystallographic results $[12,13]$ show that the subcomplex is missing the distal portion of the membrane arm. This study confirms the location of ND4 and ND5 in the distal region of the 
membrane arm, and further shows that a third proton pumping subunit, probably ND2, located proximal to the matrix arm, is still active.

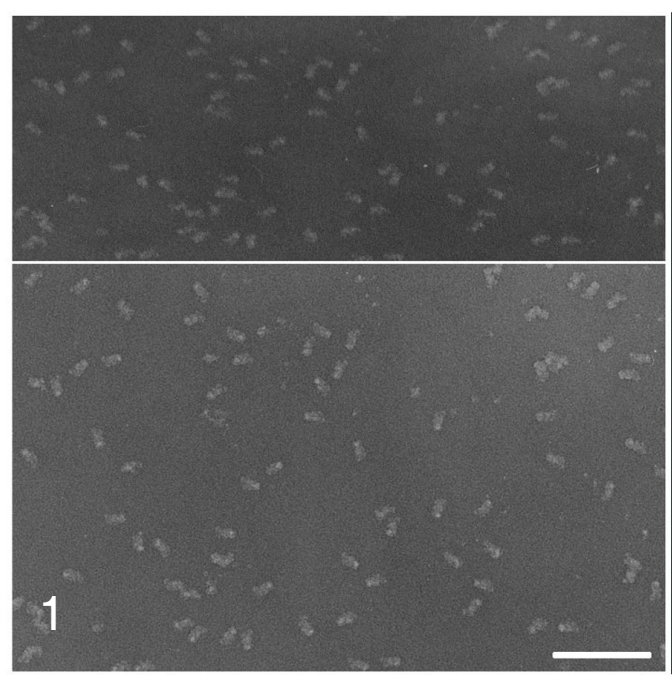

Figure 1: Tilt pair of the subcomplex. top: tilt image, bottom: $0^{\circ}$-image. Scale bar $100 \mathrm{~nm}$.

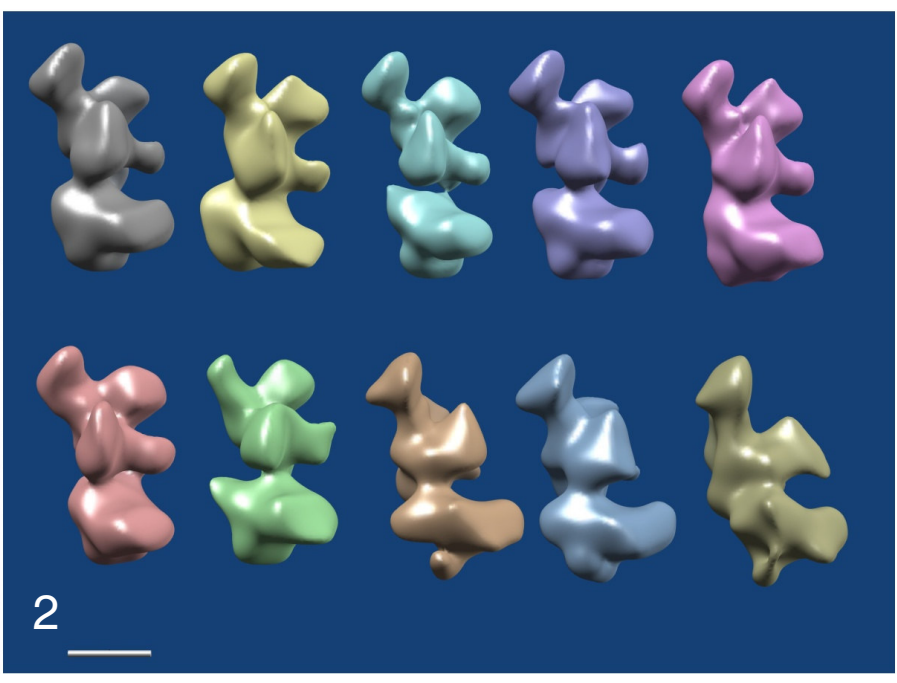

Figure 2: Ten volumes reconstructed after classification of the projections. The main difference is particle orientation. Scale bar $10 \mathrm{~nm}$.

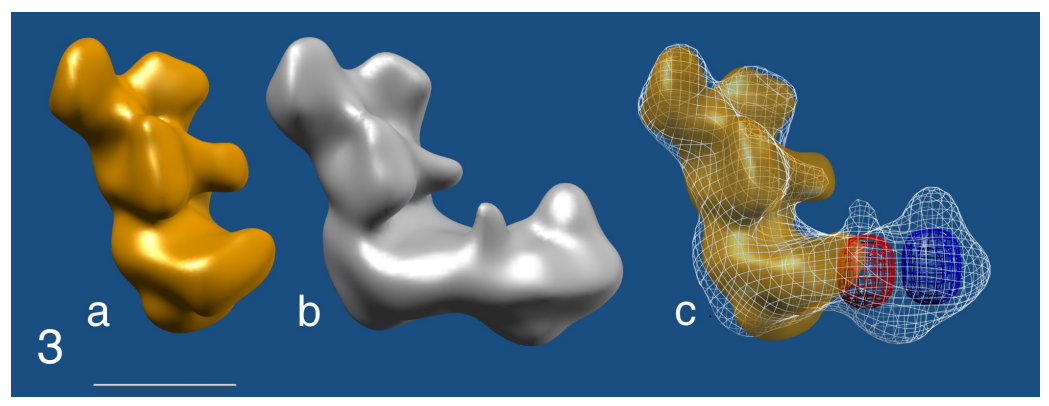

Figure 3: Comparison of the reconstructions of the subcomplex and holoenzyme. a) subcomplex, b) holoenzyme, c) overlay subcomplex/ holoenzyme. The putative locations of subunits ND4 (red) and ND5 (blue) indicated by oblongs. Scale bar $10 \mathrm{~nm}$

[1] U. Brandt, Annu. Rev. Biochem. 75 (2006) 69

[2] C. Mathiesen, C. Hägerhall, Biochim. Biophys. Acta 1556 (2002) 121

[3] E. Nakamaru-Ogiso at al., J. Biological Chemistry, 285 (2010) 39070

[4] S. Dröse et al., submitted for publication

[5] M. Radermacher et al., J. Microsc. 141 (1986) RP1

[6] T. Ruiz, M. Radermacher, Methods Mol Biol 319 (2006) 403

[7] J. K. Stoops et al., Proc. Natl. Acad. Sci. USA 89 (1992) 6585

[8] M. Radermacher, et al., J. Struct. Biol. 135 (2001) 26

[9] M. Radermacher, Ultramicroscopy 53, 1994, 121-136.

[10] P.B. Rosenthal, R. Henderson, J. Mol. Biol. 333 (2003) 721

[11] T. Clason et al., J. Struct. Biol. 169 (2010) 81

[12] C. Hunte, V. Zickermann, U. Brandt, Science 329 (2010) 448

[13] R. G. Efremov et al Nature 465, 441 (2010).

[14] Surface representations with Chimera, http://www.cgl.ucsf.edu/chimera

[15] Supported by NIH grant 2RO1 068 650, by DFG SFB815 and EXC 115 